\title{
Soutien nutritionnel des patients recevant une chimio à hautes doses avec support de cellules souches hématopoiétiques
}

\section{Par Sheryl McDiarmid}

La greffe de cellules souches hématopoïétiques (GCSH) est une thérapie intensive qui s'utilise de plus en plus pour essayer de traiter certains cancers. L'un des principaux effets indésirables de ce traitement est une absorption orale inadéquate qui peut entraîner la déshydratation et la dénutrition. Les facteurs qui peuvent causer une absorption orale inadéquate comprennent notamment les mucites, la nausée, les vomissements et l'anorexie. De plus, avant la greffe, de nombreux patients peuvent avoir éprouvé ou continué à éprouver une dénutrition associée au cancer et à son traitement.

Traditionnellement, la nutrition parentérale totale (NPT) était le soutien nutritionnel de choix pour cette population. L'équipe de greffe de moelle et de sang de l'Hôpital d'Ottawa a réduit de manière significative l'utilisation de la NPT en instituant un programme global de soutien nutritionnel qui fait appel à une gamme d'interventions dont la supplémentation orale et l'alimentation entérale.

En comprenant les causes et les répercussions de la dénutrition et en utilisant des outils qui permettent d'évaluer les risques et de mettre en œuvre, en temps opportun, les interventions nutritionnelles appropriées, on peut faciliter la guérison complète des patients parallèlement à la récupération hématopoïétique dans la population ayant subi une GCSH.

La greffe de cellules souches hématopoïétiques (GCSH) est une thérapie qui s'utilise de plus en plus pour essayer de traiter certains cancers. Les progrès réalisés dans le domaine des cellules souches et l'utilisation de facteurs de stimulation des colonies accélèrent la récupération hématopoïétique qui peut se traduire par une réduction de la durée du séjour à l'hôpital et de l'utilisation des ressources. Toutefois, la GCSH est un traitement intensif qui exige le plein emploi des interventions de soutien si on veut s'assurer que les patients, considérés dans la globalité de leur être, sont rétablis au moment de la normalisation des numérations sanguines. L'une des situations cliniques qui peut prolonger la durée du séjour ou entraîner une réadmission à la suite d'une GCSH est une prise orale insuffisante pour répondre aux besoins nutritionnels et maintenir l'état d'hydratation. En général, la durée moyenne de séjour des patients dénutris est deux fois plus longue que celle des patients bien nourris ayant des diagnostics similaires (Nixon et al., 1980; Ottery, 1995).

Certaines fonctions corporelles exigent une alimentation adéquate. Ainsi, on signale chez les patients dénutris un rétablissement différé des plaies, une réduction du taux de métabolisme des médicaments et une déficience physique et intellectuelle (Sullivan, 1995). La plupart des déficits nutritionnels inhibent les réponses immunitaires puisque les voies anabolisantes et cataboliques du système immunitaire font appel aux mêmes éléments constitutifs et sources d'énergie que d'autres activités physiologiques. La carence protéo-calorique perturbe la production d'anticorps et de complément particuliers et est une des principales causes de l'immunodéficience.

Malheureusement, il arrive qu'on n'accorde aucune priorité aux interventions de soutien nutritionnel tout au long du processus de greffe et que souvent, on attend pour les aborder que les déficiences nutritionnelles retardent le congé des patients. En vue d'assurer un état nutritionnel adéquat à la suite d'une greffe, il convient de lancer une évaluation et les interventions nutritionnelles bien avant le démarrage de la thérapie à hautes doses.

La GCSH est rarement le traitement de première ligne en ce qui concerne les cancers. La plupart des patients bénéficiant de ce traitement ont reçu au préalable des cures de chimiothérapie et de radiothérapie pour leur maladie. Fréquemment, les patients présentent déjà des signes de dénutrition lorsqu'ils arrivent au centre de greffe, et les effets toxiques de la GCSH ne font qu'exacerber la situation. La détermination de la dénutrition se base sur des mesures objectives notamment le poids, les concentrations sériques de protéines produites par le foie, les mesures anthropométriques, la force de préhension, l'anergie, les fonctions immunologiques et l'indice de masse corporelle. Aucune mesure particulière n'est suffisamment sensible pour déterminer à elle seule la dénutrition (Souba, 1997). Une dénutrition progressive perturbe le fonctionnement des organes vitaux. Toute augmentation de la sévérité de la dénutrition s'accompagne d'une augmentation de la morbidité et de la mortalité (Grant, 1992). Le moment idéal pour mettre en oeuvre une nutrition spécialisée pour les patients subissant une GCSH n'a pas été défini avec précision (Mattox, 1999). Cependant, on a constaté que le soutien nutritionnel aux personnes atteintes de cancer réduisait la morbidité et la mortalité en prévenant la perte pondérale, en augmentant la réponse au traitement, en minimisant les effets secondaires de la thérapie et en rehaussant la qualité de vie (Bloch, 1990).

Comme dans le cas des autres maladies, il est impossible de traiter la dénutrition si on n'en reconnaît pas les symptômes et si on ne pose pas le diagnostic qui s'impose. Il est essentiel d'identifier les patients à risque de dénutrition ou qui en souffrent déjà et de mettre en oeuvre un soutien nutritionnel si on prétend leur offrir des soins de première qualité (Grant, 1992). Ottery (1995) suggère que l'intervention nutritionnelle mise en place pour lutter contre la dénutrition en phase terminale a un taux de réussite potentielle aussi faible que celui des traitements chimiothérapeutiques face aux cancers en phase terminale. Les buts visant à optimaliser l'état nutritionnel doivent être établis en compagnie des patients dans le cadre de l'évaluation initiale pré-greffe.

Cet article s'intéresse au soutien nutritionnel prodigué aux patients se préparant à subir et subissant une GCSH. L'information présentée dans cet article se fonde sur une vaste recension des écrits et sur l'expérience clinique de notre établissement et d'autres centres de GCSH canadiens. La Partie une aborde les causes et les répercussions de la dénutrition chez les patients atteints d'un cancer ayant subi ou subissant actuellement une GCSH. La Partie deux, elle, passe en revue les éléments du dépistage nutritionnel et dégage les types de soutien nutritionnel disponibles. On propose également aux lectrices

Sheryl McDiarmid, RN, BScN, MEd, MBA, AOCN, ACNP, est infirmière en pratique avancée, Greffes de moelle osseuse, Hôpital d'Ottawa, Ottawa, Ontario. 
Figure un: Algorithme pour choisir parmi les options de soutien nutritionnel

Patients à faible risque (Stade A - Catégories d'évaluation globale - PG-SGA)

Le patient consomme-t-il dans son régime régulier assez de calories/jour?

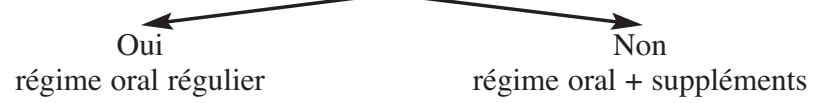

Patients à risque modéré (Stade B - Catégories d'évaluation globale - PG-SGA)

Le patient peut-il manger un régime oral adéquat + suppléments oraux?

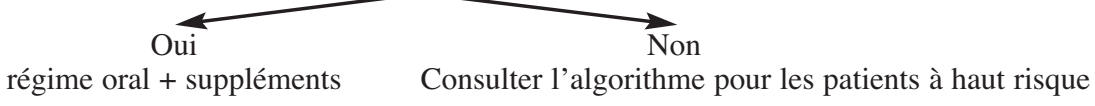

Patients à haut risque (Stade C - Catégories d'évaluation globale - PG-SGA)

Le patient peut-il manger un régime oral adéquat + suppléments oraux?

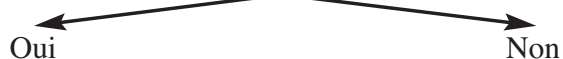

régime oral + suppléments Les intestins peuvent servir durant le traitement

Surveiller quotidiennement

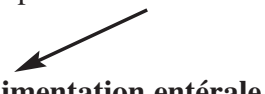

Évaluer pour alimentation entérale

Vomissements maîtrisés?

(recommencer ici si la sonde est expulsée lors d'un vomissement)

Oui

Le patient a-t-il de la mucosite?

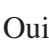

Administrer l'anti-

douleur prescrit

Administrer

l'anxiolytique prescrit

Le patient est-il thrombocytopénique?

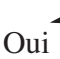

Administrer les plaquettes prescrites puis insérer la sonde naso-gastrique

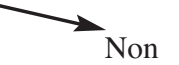

Administrer

l'anxiolytique prescrit

\section{Insérer la sonde}

naso-gastrique

Le médecin en vérifie la

position. Lancer l'alimentation

entérale selon le protocole

Résidus gastriques élevés?
Oui

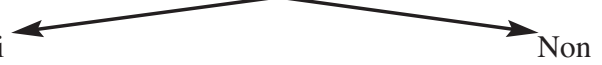

Démarrer le domperidone/la métoclopramide prescrit(e) Augmenter la dose

Diminuer la dose - Si la dose ne répond pas aux

besoins caloriques, songer à ajouter la NPT

Diarrhées en augmentation?

Oui

Enquêter sur les causes

Selles nég. pour $C$ diff:

administrer le Lomotil prescrit

Selles pos. pour $C$ diff:

administrer la Vanco prescrite

Diarrhées: en baisse/stables?

Oui

Continuer l'alimentation entérale

Continuer l'alimentation entérale

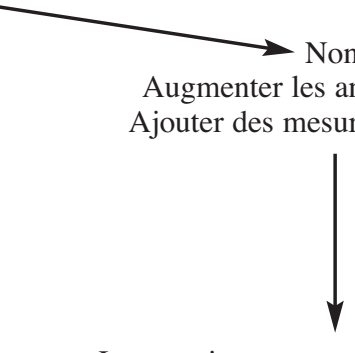

Les vomissements sont-ils maîtrisés?

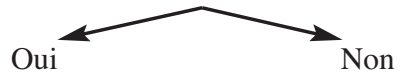

Réévaluer pour alimentation entérale

\section{Considérer la NPT}

onde d'alimentation
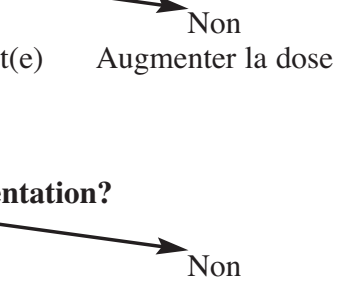
et lecteurs un algorithme permettant de choisir les interventions de soutien nutritionnel pour qu'elles soient opportunes et pertinentes (voir la figure un).

Étant donné que dans le domaine de la santé les ressources continuent de s'amenuiser, il se peut que les infirmières soient de plus en plus obligées d'assumer la responsabilité de services qui étaient dispensés jusque-là par une équipe de soutien nutritionnel. Les infirmières, fortes de l'aide des membres de l'équipe de soutien nutritionnel, occupent une position privilégiée pour surveiller l'état nutritionnel des patients, recommander des interventions et évaluer leur efficacité.

\section{Partie un: Causes choisies de la dénutrition associée à la GCSH}

La dénutrition représente le diagnostic secondaire le plus fréquent chez les patients en cancérologie (Ross, 1990). Elle est due à divers facteurs liés à la réduction de l'absorption orale, à l'augmentation des besoins métaboliques des patients, aux effets du traitement et à la tumeur proprement dite. Étant données l'intensité du traitement et la durée des symptômes, la GCSH exerce souvent des effets négatifs sur l'apport nutritionnel.

On donne le nom d'oligophagie à un apport insuffisant et elle trouve son origine dans de nombreux facteurs dont l'anorexie, la nausée, les vomissements, la dyspepsie, les mucites, l'altération du goût et des odeurs ou les aversions acquises aux aliments.

L'anorexie constitue un obstacle de taille et est fréquemment la cause première de la perte pondérale et de la cachexie. L'anorexie correspond à une diminution ou une perte d'appétit, en réponse à une sensation de faim réduite ou inexistante, malgré le besoin évident de nutriments. L'appétit est le désir psychologique de manger qui accompagne normalement les manifestations physiques de la faim telles que des tiraillements liés à la tension et à la pression existant dans la région épigastrique. L'appétit se développe dans le cadre des expériences vécues, des coutumes familiales et religieuses et c'est lui qui dicte le type d'aliments que chacun recherche de préférence. Il arrive fréquemment que l'anorexie soit la somme des autres symptômes mais elle peut également survenir sans raison connue. Les effets affectifs et psychologiques du cancer peuvent exercer un impact important sur l'appétit des patients. La présence de douleurs non maîtrisées peut altérer gravement l'appétit ainsi que l'absorption orale. Les patients anorexiques sont obligés de prendre de nombreux médicaments par voie orale et ils se plaignent qu'il n'y a pas assez de place dans leur estomac pour la nourriture et pour leurs médicaments, le tout compromettant leur absorption orale. En outre, les médicaments peuvent être anorexigènes. L'anorexie est particulièrement pénible pour les patients recevant un traitement à hautes doses car ils devraient consommer $50-70 \%$ plus de calories et deux fois plus de protéines par rapport à l'apport normal.

La nausée et les vomissements provoqués par la chimiothérapie et la radiothérapie antinéoplasiques font partie des effets secondaires les plus difficiles à contrôler (Hecht, Lembo et Chap, 1997). Les effets combinés de la chimiothérapie et de l'utilisation de l'irradiation totale lors de la GCSH provoquent des lésions tissulaires et des atteintes aux organes de grande envergure et augmentent par la suite l'incidence et l'intensité des effets gastrointestinaux. La nausée et les vomissements sont des concepts distincts, mais les termes employés pour les décrire sont souvent utilisés de manière interchangeable. Cela peut causer de la confusion et un manque de compréhension envers la prévention, l'éducation et la recherche. Rhodes, Johnson et McDaniel (1995) décrivent la nausée comme étant un phénomène subjectif et non observable d'une sensation désagréable ressentie au fond de la gorge et au niveau de l'épigastre qui peut se solder par des vomissements. On sait bien peu de choses sur la physiologie de la nausée, par rapport à celle des vomissements. La nausée précède normalement les vomissements et des doses sous-optimales d'agents émétiques peuvent à elles seules provoquer la nausée (Andrews, Rapeport et Sanger, 1988). Les patients perçoivent la nausée comme étant l'effet secondaire le plus pénible du traitement antinéoplasique (Cooper et Georgiou, 1992). Elle s'accompagne souvent des phénomènes suivants: sudation, pâleur, salivation, étourdissement, sensation vertigineuse et tachycardie. À la différence de la nausée, les vomissements peuvent se mesurer objectivement. Après que les patients ont vomi, ils déclarent souvent qu'ils se sentent mieux et que leur nausée en a été soulagée.

Les effets secondaires directement reliés à l'administration de la thérapie antinéoplasique peuvent être aggravés par des facteurs psychologiques, ce qui signifie que la nausée et les vomissements peuvent survenir à d'autres moments, la conséquence de processus d'apprentissage. On appelle ces conditions cliniques nausée et vomissements anticipés et retardés. Il peut s'avérer difficile de distinguer la nausée et les vomissements conditionnés survenant durant ou après l'administration de médicaments des effets liés aux médicaments. Malgré les améliorations apportées aux agents antiémétiques, la nausée et les vomissements anticipés et retardés continuent de représenter des effets secondaires problématiques. Morrow, Lindke et Black (1991) suggèrent qu'ils sont liés à l'utilisation de protocoles cytotoxiques plus agressifs qui annulent souvent les avances réalisées dans le domaine des antiémétiques. Étant donné que la plupart des patients bénéficiant de la GCSH ont déjà reçu des traitements de chimiothérapie, l'évaluation de la nausée et des vomissements anticipés et retardés pourrait faciliter la planification du soutien nutritionnel.

La dyspepsie est un ensemble de troubles abdominaux apparaissant après les repas. Le reflux gastro-œsophagien pathologique est le diagnostic médical mais les patients font souvent appel à des termes comme aigreurs et brûlures gastriques. Les écrits suggèrent qu'environ $10 \%$ de la population souffre de brûlures gastriques sur une base quotidienne et que plus d'un tiers des gens présentent des symptômes intermittents (Nebel, Fornes et Castell, 1976). La prévalence de la dyspepsie peut s'accroître chez les patients recevant une thérapie anticancéreuse, une conséquence de la polypharmacie qui caractérise souvent le traitement (Otto, 1994). L'évaluation de la dyspepsie est importante parce que les patients s'automédiquent fréquemment pour ce symptôme. Les interactions médicamenteuses avec les antiacides sont bien connues, et il est essentiel que les patients alertent l'équipe de soins s'ils souffrent du reflux gastro-œsophagien pathologique afin que celle-ci puisse démarrer le traitement approprié.

Des complications orales aiguës constituent un problème fréquent et souvent grave associé au reflux gastro-œsophagien pathologique. Parmi les complications les plus notoires, on remarque une inflammation et une dégradation générales des muqueuses auxquelles on donne le nom de mucites. Les protocoles de conditionnement à base de radiations et l'utilisation du Méthotrexate à des fins prophylactiques parmi les patients bénéficiant d'une allogreffe multiplient la gravité des mucites. Les douleurs buccales engendrées par les mucites peuvent également faire baisser l'absorption orale puisque les patients sont incapables d'avaler du fait même de ces douleurs. Les médicaments cytotoxiques peuvent léser les couches basales de la muqueuse orale et peuvent, de concert avec une nutrition inadéquate, amincir l'épithélium et provoquer la perte des papilles linguales dorsales. Cet épithélium atrophié est incapable de résister à l'usure normale associée à l'alimentation et à la parole.

L'oligophagie peut elle aussi être causée par des facteurs indirects tels que des altérations du goût et de l'odorat. Il convient d'effectuer une évaluation de la bouche chez tous les patients. 
Beaucoup de patients atteints de cancer se plaignent de l'altération du goût et cette dernière peut être d'une gravité telle qu'elle en perturbe la nutrition (Wickham et al., 1999). La sensibilité des papilles gustatives peut être modifiée par les changements chroniques touchant leur environnement immédiat comme une quantité moindre de salive et/ou la candidose. Une mauvaise hygiène orale perturbe le goût en permettant à une couche de débris de recouvrir les papilles gustatives qui entraîne la fatigue gustative. Ces débris en décomposition peuvent également entraver la détection des saveurs (Otto, 1994). Des goûts désagréables peuvent causer nausée et vomissements; on ne sait pas exactement s'il s'agit d'une réaction primaire ou d'une réaction secondaire résultant d'une exposition préalable. Les patients souffrant d'une altération du goût peuvent présenter une diminution de l'apport calorique et une augmentation de l'indice de perte pondérale par rapport aux patients dont le goût est resté normal (DeWys et Pascucci-Cimino, 1978). On sait que des médicaments tels que les antibiotiques, les antifongiques, les narcotiques et certains agents chimiothérapeutiques contribuent à l'altération du goût. Chez certains patients, la sécheresse de la bouche est due à la baisse des sécrétions salivaires et elle est habituellement secondaire à la radiothérapie. Elle s'aggrave s'il leur arrive de souffrir de déshydratation ou de fièvre. Quand on manque de salive, il est pénible de mastiquer et d'avaler un grand nombre d'aliments.

Les aversions alimentaires acquises qui résultent de l'association entre certains aliments et des symptômes désagréables peuvent favoriser l'oligophagie. On estime que l'incidence de cette dernière dans la population générale en bonne santé se situe entre $38 \%$ et 65 $\%$ (Mattes, Arnold et Boraas, 1987). Afin de prévenir ce syndrome, on demandait habituellement aux patients d'éviter de manger de grandes quantités de nourriture avant tout traitement comportant un médicament susceptible de causer la nausée et des vomissements. Des études montrent désormais que les aversions alimentaires peuvent se former à tout moment dans les 48 heures précédant ou suivant le premier traitement. Il n'est pas raisonnable de demander aux patients d'éviter de manger pendant quatre jours en vue d'abaisser le risque de développer des aversions alimentaires. Ce mécanisme peut également entraîner des aversions aux suppléments nutritionnels. Il est possible que des suppléments nutritionnels aient été prescrits en vue d'optimaliser l'absorption orale avant la GCSH; il importe donc de determiner si des suppléments sont nécessaires et quels types on pourra choisir au besoin.

De nombreuses variables contribuent au risque de dénutrition chez les patients bénéficiant d'une GCSH. Le degré de soutien nutritionnel dépend des buts globaux des patients et de l'équipe de soins. La GCSH étant à visée curative, il convient de maximiser les ressources de soutien afin d'augmenter les retombées du traitement. Les interventions de soutien nutritionnel doivent donc constituer une stratégie en vue d'optimaliser la réponse au traitement et de favoriser le rétablissement post-GCSH.

\section{Partie deux: Dépistage nutritionnel et interventions de soutien nutritionnel}

Lorsqu'on planifie les soins nutritionnels dans le cadre de la GCSH, il est essentiel de mettre sur pied une évaluation nutritionnelle et un programme de soutien nutritionnel personnalisés (Vaughn et McTierman, 1986). Bien que l'évaluation de l'état nutritionnel soit difficile et qu'aucun indicateur individuel ne semble valide de manière isolée (Reilly, Martineau, Moran et Kennedy, 1995), de nombreux outils d'évaluation sont disponibles. Il faut disposer d'une évaluation rapide et valide, et l'outil de la Dre Faith Ottery, dont la version française s'intitule Auto-évaluation nutritionnelle globale subjective (PG-SGA), a fait l'objet d'une élaboration et d'une validation de qualité (Ottery, 2000). Cet outil prend environ cinq minutes à compléter. Le patient remplit la première partie du formulaire (historique du poids, prise alimentaire, symptômes et capacité fonctionnelle). Ensuite, la clinicienne complète le reste du formulaire en fonction des résultats de l'examen physique. Le score de la PG-SGA s'obtient en ajoutant le score du patient à celui de la clinicienne et en plaçant les patients dans l'une des trois catégories suivantes: Bien nourri (Stade A), Modérement dénutri (Stade B), ou Sévèrement dénutri (Stade C). Le tableau un résume les catégories d'évaluation globale de la PG-SGA. Une fois cette évaluation

Tableau un: PG - SGA: Catégories d'évaluation globale

\begin{tabular}{|c|c|c|c|}
\hline & Stade A & Stade B & Stade C \\
\hline Catégorie & Bien nourri & $\begin{array}{l}\text { Modérément dénutri ou } \\
\text { dénutrition soupçonnée }\end{array}$ & Sévèrement dénutri \\
\hline Poids & $\begin{array}{l}\text { Aucune perte pondérale ou récent } \\
\text { gain pondéral non liquide }\end{array}$ & $\begin{array}{l}\text { Perte pondérale de } 5 \% \text { en } \\
\text { l'espace d'1 mois (ou } 10 \% \text { en } \\
\text { l'espace de } 6 \text { ) } \\
\text { Aucune stabilisation pondérale ni } \\
\text { aucun gain pondéral (perte } \\
\text { pondérale continue) }\end{array}$ & $\begin{array}{l}\text { Perte pondérale sup. à } 5 \% \text { en l'espace } \\
\text { d'1 mois (ou à } 10 \% \text { en l'espace de } 6 \text { ) } \\
\text { Aucune stabilisation pondérale ni aucun } \\
\text { gain pondéral (perte pondérale continue) }\end{array}$ \\
\hline $\begin{array}{l}\text { Apport en } \\
\text { nutriments }\end{array}$ & $\begin{array}{l}\text { Ni déficit ni amélioration } \\
\text { significative récente }\end{array}$ & Diminution évidente de l'apport & Grave déficit au niveau de l'apport \\
\hline $\begin{array}{l}\text { Symptômes à } \\
\text { impact d'ordre } \\
\text { nutritionnel }\end{array}$ & $\begin{array}{l}\text { Aucun symptôme ou amélioration } \\
\text { significative récente permettant } \\
\text { l'apport adéquat }\end{array}$ & $\begin{array}{l}\text { Présence de symptômes à impact } \\
\text { d'ordre nutritionnel } \\
\text { (vomissements, diarrhée, } \\
\text { nausée, mucosite) }\end{array}$ & $\begin{array}{l}\text { Présence de symptômes à impact d'ordre } \\
\text { nutritionnel (vomissements, diarrhée, } \\
\text { nausée, mucosite) }\end{array}$ \\
\hline Fonctionnement & $\begin{array}{l}\text { Aucun déficit ou amélioration } \\
\text { significative récente }\end{array}$ & $\begin{array}{l}\text { Déficit fonctionnel modéré ou } \\
\text { détérioration récente }\end{array}$ & $\begin{array}{l}\text { Déficit fonctionnel sévère ou détérioration } \\
\text { récente significative }\end{array}$ \\
\hline $\begin{array}{l}\text { Examen } \\
\text { physique }\end{array}$ & $\begin{array}{l}\text { Aucun déficit ou déficit chronique } \\
\text { présentant une amélioration } \\
\text { clinique récente }\end{array}$ & $\begin{array}{l}\text { Constatation d'une perte légère à } \\
\text { modérée de tissu adipeux } \\
\text { sous-cutané et/ou de la masse } \\
\text { musculaire et/ou du tonus } \\
\text { musculaire à la palpation }\end{array}$ & $\begin{array}{l}\text { Signes de dénutrition évidents } \\
\text { (p. ex. perte sévère de tissus sous-cutanés, } \\
\text { possibilité d'œdème) }\end{array}$ \\
\hline
\end{tabular}


effectuée, il est essentiel que l'information recueillie serve à élaborer un plan impliquant tous les membres de l'équipe soignante qui sera ensuite mis en oeuvre, évalué et révisé au besoin.

Bien que certains patients candidats à la GCSH commencent leur cheminement dans la catégorie Bien nourri, de nombreux patients en sont au Stade B ou C lorsqu'ils se présentent au centre de greffe à des fins d'évaluation. Tous les patients courent le risque de passer aux catégories Modérément dénutri et Sévèrement dénutri et c'est seulement en mettant en oeuvre de manière adéquate l'évaluation initiale, l'intervention et l'évaluation de cette dernière qu'on optimisera la nutrition chez les patients bénéficiant de la GCSH.

Maintenant que l'on dispose de toute cette information, comment décide-t-on des interventions de soutien nutritionnel qu'il convient de recommander en vue de fournir une nutrition adéquate et de maintenir ou d'améliorer l'état nutritionnel? La prochaine section traitera de trois interventions de soutien nutritionnel: supplémentation orale, alimentation entérale et nutrition parentérale totale.

\section{Supplémentation orale}

La devise associée à la supplémentation orale devrait être "il faut que chaque bouchée compte". L'optimalisation de l'absorption orale chez les patients hospitalisés est un défi de taille, il est donc essentiel de sensibiliser les patients à l'importance d'une nutrition adéquate au cours de la phase précédant la GCSH, lorsqu'ils passent la majeure partie de leur temps en dehors de l'hôpital. Les membres du personnel soignant sont nombreux à supposer que les gens connaissent bien les principes d'une bonne nutrition; c'est pourtant à ce moment-là qu'il faut aborder les principes fondamentaux de la nutrition. Les patients sont tellement fascinés par les interventions technologiques qu'ils minimisent l'impact de saines pratiques de préservation de la santé comme l'apport nutritionnel. La distribution d'une documentation écrite renfermant de sains conseils nutritionnels, à laquelle on ajoutera une consultation diététique, pourra aider les patients à choisir les interventions nutritionnelles appropriées à leurs troubles alimentaires particuliers. On pourra faire appel, entre autres, aux interventions suivantes: modification de la texture des aliments, repas et collations prises à des heures régulières, augmentation de la densité nutritionnelle des aliments et utilisation d'aliments et/ou de suppléments riches en calories et en protéines.

La majorité des cliniciens connaissent bien les suppléments du commerce mis à la disposition des patients dans les hôpitaux. Toutefois, les suppléments faits maison peuvent avoir les mêmes propriétés nutritionnelles et il convient d'en encourager la consommation afin de varier le "menu". La diététiste peut fournir aux patients et aux proches l'information dont ils ont besoin pour préparer leurs propres suppléments. Puisqu'une partie toujours plus grande des soins est dispensée en clinique externe, les interventions qui peuvent être personnalisées pour être effectuées au foyer revêtent une importance essentielle. Dans notre établissement, quarante pour cent de la durée totale du séjour correspondant au processus de la GCSH concernent les soins en clinique externe.

Lorsque la prise alimentaire orale devient insuffisante, il peut être nécessaire de compléter l'apport oral pour qu'il satisfasse aux besoins caloriques. Deux techniques de supplémentation nutritionnelle peuvent être alors considérées: l'alimentation entérale et la nutrition parentérale totale (NPT).

\section{Alimentation entérale}

L'alimentation entérale est la méthode qui consiste à faire parvenir une solution nutritive dans la voie gastro-intestinale au moyen d'une sonde. On peut se servir des sondes suivantes: sonde naso-gastrique (NG) ou naso-intestinale (NI), sonde de gastrostomie ou de jéjunostomie. L'emploi de l'alimentation entérale a augmenté grâce aux améliorations apportées aux formules et aux systèmes d'administration et parce qu'aujourd'hui, les cliniciens apprécient mieux ses avantages. Lorsqu'elle est possible, l'utilisation de l'appareil gastro-intestinal préserve la fonction gastro-intestinale et minimise les infections reliées aux entéropathogènes. La devise de l'alimentation entérale devrait être "si les intestins fonctionnent, utilisons-les" (Kaminski et Blumeyer, 1993). On croyait jadis que la voie gastro-intestinale jouait un rôle passif au niveau de l'absorption des nutriments et qu'elle fonctionnait de manière sous-optimale chez les malades en phase critique et qu'on pouvait facilement la contourner en faisant appel à l'alimentation parentérale. Il appert maintenant que les intestins jouent un rôle central dans la réponse aux atteintes et que le maintien de la fonction intestinale normale grâce à l'alimentation entérale et à l'apport des nutriments essentiels peut accélérer le rétablissement et améliorer la survie (Heinburger et Weinser, 1997; Wilmore, Smith et Odwyer, 1988).

La translocation bactérienne est le mouvement des bactéries et des endotoxines qui colonisent normalement les intestins à travers la muqueuse intestinale, mouvement qui se solde par une invasion d'organes et de tissus. Une déficience des défenses immunitaires de l'hôte et des atteintes à la muqueuse intestinale, qui accompagnent communément la thérapie à hautes doses, facilitent la translocation bactérienne. La présence d'aliments dans les intestins aide à inhiber la pénétration de la muqueuse par des pathogènes. Ces aliments stimulent la production de sécrétions qui aident à préserver la fonction barrière de la voie gastro-intestinale et stimulent la régénération continue des cellules épithéliales, le péristaltisme et la défécation qui, à leur tour, réduisent l'exposition de la muqueuse aux pathogènes et sa colonisation ultérieure (Phillips et Olsen, 1993).

Les contre-indications à l'alimentation entérale sont au nombre de quatre: occlusion intestinale, état de choc, résection de l'intestin $>90 \%$ et insuffisance mésentérique artérielle (ASPEN, 1987). À moins qu'elle ne soit contre-indiquée, il est conseillé de démarrer l'alimentation entérale dès que les patients ne sont plus capables de répondre à leurs besoins nutritionnels quotidiens par le biais de l'absorption orale régulière, y compris la supplémentation.

\section{Raisons de l'échec de l'alimentation entérale}

L'attitude négative de membres de l'équipe soignante et de patients constitue l'un des principaux obstacles à l'alimentation entérale. On peut le surmonter en insistant sur le concept du soutien nutritionnel optimal. Il peut sembler plus aisé de recevoir son alimentation par le biais d'un cathéter veineux central, mais cette option ne constitue pas le soutien nutritionnel optimal pour bon nombre de patients. Les membres de l'équipe soignante doivent faire preuve de cohérence dans leur approche du soutien nutritionnel. Le rôle de la nutrition entérale doit avoir une valeur similaire au choix d'antibiotique ou de régime de chimiothérapie. Une consultation précoce sur le soutien nutritionnel et la distribution d'une documentation écrite soulignant la philosophie $\mathrm{du}$ soutien nutritionnel peuvent mettre l'accent sur les avantages de l'alimentation entérale en ce qui a trait à l'optimalisation des soins aux patients et à leur rétablissement. Les infirmières peuvent aider les patient à s'adapter aux changements relatifs à l'image corporelle en leur dispensant leur enseignement, en leur offrant un soutien affectif et en manifestant une attitude compréhensive.

Les autres raisons derrière l'échec de l'alimentation entérale comprennent la nausée et les vomissements qui peuvent entraîner l'expulsion de la sonde d'alimentation, des diarrhées et une forte quantité de résidus gastriques. Il convient de réaliser l'évaluation des symptômes décrits par les patients en vue d'évaluer la réussite de l'alimentation entérale. Il se peut que les infirmières, les 
médecins, les diététistes et les patients voient dans la nausée et les vomissements un seul et même symptôme et qu'ils soient donc peu enclins à continuer l'alimentation entérale. Comme nous l'avons signalé auparavant, un plus grand nombre de patients éprouvent de la nausée plutôt que de véritables vomissements, et il peut s'écouler de longs intervalles entre les épisodes de vomissements (Cooper et Georgiou, 1992). La nausée ne constitue pas nécessairement un élément dissuasif à l'alimentation entérale. Les vomissements non plus d'ailleurs. Gulley, Vander Pleog et Gulley (1993) ont décrit un protocole de traitement pour l'hyperémèse qui distribue en infusion continue un produit d'alimentation par sonde iso-osmolaire. Ce groupe utilisait la voie de l'alimentation entérale pour traiter les symptômes qui interdisent traditionnellement l'emploi de l'alimentation entérale chez les patients en cancérologie. Si, mis à part les vomissements et l'expulsion de la sonde d'alimentation, l'alimentation entérale donne de bons résultats, ces problèmes ne doivent pas à eux seuls constituer le seul critère d'arrêt du protocole. Notre expérience montre que les patients à qui l'on a enseigné l'importance de la nutrition optimale ne s'opposent pas à la réinsertion de la sonde d'alimentation.

La diarrhée constitue une complication fréquente de la thérapie à hautes doses et il est donc difficile d'en déterminer le facteur causal. Thomas (1994) suggère que le type de formule d'alimentation par sonde est rarement à l'origine de la diarrhée et qu'il est inutile d'arrêter la formule d'alimentation par sonde en attendant que la cause de la diarrhée soit identifiée. Des divers mécanismes de la diarrhée, la diarrhée osmotique est une cause relativement fréquente (Greenwood, 1994). La principale cause de la diarrhée osmotique est l'administration de médicaments qui augmentent la charge osmotique. Bien qu'il puisse paraître avantageux d'administrer les médicaments au moyen de la sonde d'alimentation, il importe d'évaluer l'osmolalité et/ou le contenu en sorbitol de chaque médicament en vue de dégager sa susceptibilité de causer la diarrhée. Les médicaments auxquels Thomas renvoie incluent notamment les antiacides à base de magnésium, la cimetidine, les suppléments à base de potassium et de phosphore, les composés de la quinidine, le lactulose et d'autres laxatifs. Bon nombre d'élixirs renferment du sorbitol, fréquemment à des doses laxatives qui provoquent la diarrhée en stimulant la motilité gastro-intestinale et en augmentant le processus sécrétoire. Si on soupçonne le facteur contributif des médicaments, il est peut-être indiqué de passer à une formule sans élixir ou à l'administration parentérale.

La diarrhée peut se présenter chez les patients recevant des antibiotiques étant donné que ceux-ci altèrent la colonisation de la voie gastro-intestinale. Ceci se produit que les patients soient alimentés par voie entérale ou par voie parentérale. En revanche, des diarrhées sévères peuvent se traduire par la malabsorption des nutriments, et la nutrition parentérale pourrait alors être indiquée.

La réaction du greffon contre l'hôte est une complication de l'allogreffe et elle se manifeste au niveau de la peau, du foie et/ou de la voie gastro-intestinale. L'incidence de la réaction aiguë du greffon contre l'hôte, qui survient au cours des 100 premiers jours suivant la greffe, varie de 20 à $80 \%$ selon le degré d' histocompatibilité, le nombre de lymphocytes $\mathrm{T}$ dans le greffon, l'âge des patients et le traitement immunosuppresseur préventif utilisé. Au niveau de la voie gastro-intestinale, la réaction du greffon contre l'hôte peut se manifester par des diarrhées avec ou sans nausée et vomissements, des douleurs abdominales et/ou un iléus. La diarrhée associée à la réaction du greffon contre l'hôte a une apparence caractéristique d'un vert aqueux. Le traitement de la réaction du greffon contre l'hôte au niveau des intestins comprend les corticostéroïdes et le repos intestinal jusqu'à ce que les symptômes s'amenuisent. Ceci écarte la possibilité de faire appel à l'alimentation entérale durant la phase aiguë.
Une autre complication des greffes, principalement en ce qui concerne les allogreffes, est la maladie veino-occlusive du foie (MVOF). Il s'agit d'un syndrome clinique qui se caractérise par une hépatomégalie douloureuse, une rétention de fluides et l'hyperbilirubinémie. Du point de vue histologique, la MVOF se définit comme étant le rétrécissement concentrique progressif des veinules intrahépatiques, associé à la nécrose des hépatocytes. Cependant, on ne comprend pas encore entièrement les mécanismes et les phénomènes cellulaires à l'œuvre dans la MVOF. Le diagnostic clinique de la MVOF se fonde sur la présence de jaunisse, d'hépatomégalie et/ou de douleurs et d'une ascite dans le quadrant supérieur droit et/ou d'une prise de poids inexpliquée. Les écrits ne signalent aucune étude où l'alimentation entérale était utilisée chez ce groupe de patients. L'utilisation de la nutrition parentérale totale est limitée chez ces patients-là du fait de la toxicité connue pour le foie de l'emploi de la NPT et de la nécessité de restreindre l'apport liquidien.

La complication la plus dangereuse de l'alimentation entérale est l'aspiration du produit alimentaire dans les poumons (Burchett, 1994). En plus d'évaluer l'absorption, il faut veiller à ce que les résidus gastriques restent minimes pour réduire le risque d'aspiration si le patient devait vomir. Pour prévenir l'aspiration, on soulève la tête du lit d'au moins 30 degrés pendant les séances d'alimentation et durant l'heure qui suit (Bowers, 2000). La vidange gastrique peut être retardée par la septicémie, une glycémie élevée, la gastroparésie diabétique, une température élevée, l'ischémie ou des réparations chirurgicales et la teneur en matière grasse de la formule (Thomas, 1994). L'alimentation gastrique convient aux patients dont le réflexe nauséeux et le réflexe tussigène sont intacts et chez qui la vidange gastrique reste adéquate. Les sondes naso-duodénales et naso-jéjunales sont généralement indiquées pour les patients en phase critique présentant un risque d'aspiration pulmonaire ou de vidange gastrique retardée (Bowers).

On présente, à titre d'outil de prise de décision, un algorithme facilitant le démarrage des différentes options de soutien nutritionnel (voir la figure un). Puisque les protocoles d'alimentation varient, il faut connaître la politique de son établissement relative à l'introduction de l'alimentation entérale, les soins entourant les sondes d'alimentation et leur entretien et les lignes directrices guidant l'évaluation et l'intervention infirmières.

\section{Nutrition parentérale totale}

La nutrition parentérale totale (NPT) représentait le soutien nutritionnel de choix pour les patients subissant une GCSH (Herrmann et Petruska, 1993). Quoique des études aient montré que la plupart des patients bénéficiant d'une allogreffe nécessitent une forme ou une autre de NPT durant la période post-greffe, la NPT n'a pas connu des résultats uniformes en ce qui a trait à l'amélioration de l'état nutritionnel ou des résultats pour le patient (Szeluga, Stuart, Brookmeyer, Utermohlen et Santos, 1987; Weisdorf, Hofland et Sharp, 1984).

La NPT se présente sous la forme d'une solution renfermant du glucose, des acides aminés, des vitamines, des minéraux et oligoéléments et des lipides. Elle s'administre souvent au moyen de cathéters veineux centraux. Bien que la NPT soit parfois une mesure nécessaire à la survie des patients incapables de tolérer un régime entéral, elle n'est pas sans risques. Les patients qui reçoivent la NPT subissent un plus grand nombre de complications techniques et métaboliques comme un risque d'infection plus élevé, notamment pour ce qui est des infections fongiques (Hermann et Petruska, 1993). Il ne faut donc pas utiliser la NPT à tort et à travers, surtout chez les patients dont les intestins fonctionnent; il existe pourtant deux recommandations standards pour la mise en oeuvre de la NPT chez cette population: (1) 
lorsqu'on s'attend à ce que le traitement dure de cinq à sept jours à cause d'effets gastro-intestinaux secondaires, et (2) lorsque les patients peuvent consommer, par la voie orale ou entérale, seulement $60 \%$ de leurs besoins nutritionnels sur une période de trois à cinq jours (Mattox, 1999). En cas de réaction aiguë du greffon contre l'hôte, le soutien nutritif est d'une importance capitale si on veut favoriser la réparation des tissus; comme nous l'avons signalé auparavant, lorsque la réaction aiguë touche les intestins, on pourra faire appel à la NPT en vue de promouvoir le repos intestinal. Dans notre établissement, plusieurs patients ont dû subir une NPT à long terme afin de satisfaire à leurs besoins nutritionnels tandis que leurs intestins se rétablissaient de la réaction du greffon contre l'hôte. L'Hôpital d'Ottawa a mis sur pied un programme qui enseigne aux patients à réaliser leurs propres infusions de NPT à domicile. Un protocole de concentration de la NPT permet de l'administrer la nuit en l'espace de huit-12 heures, et les patients peuvent ainsi se livrer à leurs activités quotidiennes qui seraient nécessairement limitées par des infusions continues. Ce protocole s'est révélé essentiel au maintien en vie d'un bon nombre de patients.

\section{Alimentation de transition}

Tout comme les autres interventions thérapeutiques, le soutien nutritionnel exige une évaluation permanente et une modification ultérieure de la thérapie. L'utilisation d'une forme particulière de soutien nutritionnel ne doit pas nécessairement exclure les autres et les patients peuvent bénéficier d'une association d'approches. Une évaluation permanente est de rigueur lorsqu'on soigne des patients ayant bénéficié d'une GCSH puisque les symptômes peuvent changer rapidement. Tel patient pourra développer une mucosite sévère du jour au lendemain, ce qui nécessitera une modification de la texture de la nourriture ou encore le démarrage de l'alimentation entérale. L'évolution de la situation peut également se faire dans l'autre sens. Nous avons découvert, qu'avec l'emploi de cellules souches périphériques et une fois que la numération des neutrophiles s'est rétablie, la mucosite peut s'améliorer de manière spectaculaire en l'espace de 24 heures et on peut donc recommencer l'absorption orale avec l'objectif de mettre fin à l'alimentation entérale.

Le passage d'une voie d'alimentation à une autre ou d'une formule à une autre s'appelle alimentation de transition. Étant donné le coût élevé de la NPT et ses éventuelles complications métaboliques et mécaniques, il est fort souhaitable d'effectuer la transition de la NPT à la nutrition entérale ou à la nutrition orale dès que cela s'avère médicalement possible. Les candidats à l'alimentation de transition sont les patients qui présentent une voie gastro-intestinale viable et des signes de tolérance gastrointestinale. La structure et le fonctionnement de la muqueuse intestinale profitent des plus petites quantités de nutriments fournis par voie orale. La figure deux présente un algorithme permettant de déterminer la transition de la nutrition parentérale à la nutrition entérale et orale.

Les écrits portant sur le rôle de l'alimentation entérale dans la GCSH ne sont pas légion. La plupart des patients recevant une thérapie à hautes doses vont continuer à prendre, par la voie orale, des aliments et des liquides en quantité variables, et il faut toujours les encourager à accroître leur absorption orale. Une fois que les besoins nutritionnels des patients sont satisfaits grâce à l'absorption orale, on peut leur donner l'alimentation entérale à titre cyclique. Normalement, on l'administre la nuit et on pense qu'elle accroît l'appétit et l'ambulation des patients durant le jour (Trujillo et Queen, 1994).

Les apports nutritionnel et liquidien doivent être surveillés quotidiennement. L'absorption orale peut progresser tandis que le patient continue de recevoir une alimentation par sonde à domicile.
On peut enseigner aux patients et aux proches les techniques d'administration de l'alimentation entérale à domicile. Ceci abrège la durée de l'hospitalisation puisque les patients peuvent augmenter graduellement leur absorption orale en faisant aussi appel à l'alimentation entérale à titre de supplémentation nutritionnelle. On pourra retirer la sonde une fois que le patient consomme régulièrement des $2 / 3$ aux $3 / 4$ de ses besoins protéo-energétiques sur une période de trois jours.

La nutrition entérale gagne en popularité à mesure que des études mettent en évidence les effets bénéfiques du soutien nutritionnel administré directement au niveau des intestins, l'utilisation améliorée des substrats fournis par l'alimentation entérale et les avantages économiques (Johansson, Backman et Jakobsson, 1996; Mainous, Block et Deitch, 1994). Toutefois, de nouvelles études devront être menées au sein de la population de patients bénéficiant de la GCSH afin de déterminer à la fois les patients les plus susceptibles de bénéficier de l'alimentation entérale et du moment idéal pour démarrer ce type d'alimentation.

\section{Répercussions pour la pratique infirmière}

Les infirmières occupent une position privilégiée pour aider les patients et les familles dans le domaine de la nutrition. Il existe une foule d'options de mise en œuvre des interventions de soutien nutritionnel aux patients subissant une GCSH, mais la meilleure stratégie pour chaque patient consiste toujours à effectuer une évaluation en profondeur des facteurs contributifs en recueillant, en plus, l'opinion de l'équipe multidisciplinaire. L'évaluation infirmière et la gestion des symptômes des patients contribuant à la réduction de l'absorption orale et à la perte de nutriments sont des éléments clés de la réussite de toute intervention. La gestion de la nausée, des vomissements et des diarrhées exige d'excellentes compétences en évaluation et une connaissance des actions pharmacologiques. Une évaluation précise de la douleur suivie de l'administration des analgésiques appropriés pourra aider les patients à optimiser leur absorption orale. En faisant la promotion de l'hygiène orale et en aidant les patients à l'accomplir, on minimise non seulement le risque d'introduction de bactéries dans la cavité buccale, mais on les aide également à préserver l'intégrité de la muqueuse orale qui joue un rôle capital dans le rétablissement d'une nutrition orale adéquate.

\section{Figure deux: Algorithme concernant la transition de la nutrition parentérale}

Le patient reçoit la nutrition parentérale

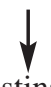

La voie gastro-intestinale fonctionne-t-elle correctement pour permettre l'absorption des nutriments?

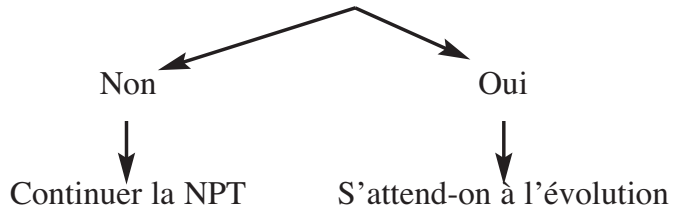
rapide de l'absorption orale adéquate?

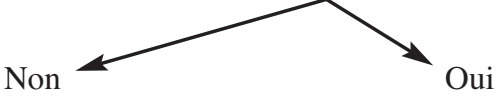

Lancer l'alimentation entérale Régime oral +/supplémentation 
Les infirmières peuvent aussi fournir leur appui aux proches qui éprouvent souvent de la frustration lorsqu'ils sont incapables d'améliorer l'absorption orale des patients. C'est cette frustration qui les amène à exercer des pressions sur l'équipe soignante pour qu'elle mette en route la NPT. Les infirmières peuvent mettre l'accent sur les avantages de la nutrition orale et aider les patients et leur famille à établir des buts et à sélectionner les interventions qui répondront aux besoins à court et à long terme.

En termes de recherche, cet article présente un survol des causes de la dénutrition chez les patients subissant une GCSH et il passe en revue les types de soutien nutritionnel disponibles. Il appert cependant que de nouvelles recherches sont nécessaires dans divers domaines liés à la supplémentation nutritionnelle chez cette population. Bien que cela fasse huit ans que l'Hôpital d'Ottawa utilise l'alimentation entérale à titre de stratégie de soutien nutritionnel chez les patients bénéficiant d'une GCSH, on n'a pas encore évalué l'impact de ces interventions sur les résultats pour les patients. Il est légitime de réclamer des recherches dont les données probantes serviront à orienter la pratique dans le domaine du soutien nutritionnel.

\section{Références}

Andrews, P.L.R., Rapeport, W.G., \& Sanger, G. (1988). Neuropharmacology of emesis induced by anticancer therapy. Trends in Pharmacology Science, 9, 334-41.

ASPEN Board of Directors. (1987). Guidelines for the use of enteral nutrition in the adult patient. JPEN, 11, 435-439.

Bloch, A. (1990). Nutrition Management of the Cancer Patient. Rockland, ML: Aspen.

Bowers, S. (2000). All about tubes: Your guide to enteral feeding devices. Nursing 2000, 30(12), 41-48.

Burchett, H. (1994). Nursing care of the tube-fed patient. Nutritionall, 2(1), 6-7.

Cooper, S., \& Georgiou, V. (1992). The impact of cytotoxic chemotherapy - Perspectives from patients, specialists and nurses. European Journal of Cancer, 28A, 536-38.

DeWys, W.D., \& Pascucci-Cimino, M. (1978). Anorexia, taste changes and diet in cancer. Comprehensive Therapy, 4, 7-12.

Grant, J.P. (1992). Handbook of Total Parenteral Nutrition (2nd ed.). Philadelphia, PA: Saunders.

Greenwood, J. (1994). Diarrhea in tube-fed patients. Nutritionall, 2(1), 4-5.

Gulley, R.M., Vander Pleog N., \& Gulley, J.M. (1993). Treatment of hyperemesis gravidarum with nasogastric feeding. Nutrition in Clinical Practice, 8(1), 33-35.

Hecht, J.R., Lembo, T., \& Chap, L. (1997). Prolonged nausea and vomiting after high dose chemotherapy and autologous peripheral stem cell transplantation in the treatment of high risk breast carcinoma. Cancer, 79, 1698-1702.

Heinburger, D.C., \& Weinser, R.L. (1997). Handbook of Clinical Nutrition (3rd ed.). St. Louis, MO: Mosby.

Herrmann, V.M., \& Petruska, F.J. (1993). Nutritional support in BMT recipients. Nutrition in Clinical Practice, 8(1), 19-27.

Johansson, C., Backman, L., \& Jakobsson, L. (1996). Is enteral nutrition used in hospital patients: A study of the practice of nutrition in a Swedish hospital. Clinical Nutrition, 15, 171-174.

Kaminski, M., \& Blumeyer, T. (1993). Metabolic and nutritional support of the intensive care patient: Ascending the learning curve. Critical Care Clinics, 9(2), 363-76.

Mainous, M., Block, E., \& Deitch, E. (1994). Nutritional support of the gut, how and why. New Horizons, 2(2), 193-210.

Mattes, R.D., Arnold, C., \& Boraas, M. (1987). Management of learned food aversion in cancer patients receiving chemotherapy. Cancer Treatment Reports, 71, 1071-78.

Mattox, T.W. (1999). Specialized nutrition management of patients receiving hematopoietic stem cell transplantation. Nutrition in Clinical Practice, 14, 5-15.

Morrow, G.R., Lindke, J., \& Black, P.M. (1991). Predicting development of anticipatory nausea in cancer patients: Prospective examination of eight clinical characteristics. Journal of Pain \& Symptom Management, 6(4), 215-23.

Nebel, O.T., Fornes, M.F., \& Castell, D.O. (1976). Symptomatic gastroesophageal reflux: Incidence and precipitating factors. American Journal of Digestive Diseases, 21, 953-956.
Nixon, D.W., Heymsfield, S.B., Cohen, A.E., Kutner, M.H., Ansley, J., Lawson, D.H., \& Rudman, D. (1980). Protein calorie undernutrition in hospitalized cancer patients. American Journal of Medicine, 68,683 .

Ottery, F.D. (1995). Supportive nutrition to prevent cachexia and improve quality of life. Seminars in Oncology Nursing, 22(2), S3, 98-111.

Ottery, F.D. (2000). Patient-generated subjective global assessment. In P.D. McCallum \& C.G. Polisena (Eds.), The clinical guide to oncology nutrition (pp. 236-252). Chicago: American Dietetic Society.

Otto, S. (1994). Oncology Nursing. St. Louis, MO: Mosby.

Phillips, M., \& Olsen, L. (1993). The immunologic role of the gastrointestinal tract. Critical Care Nursing Clinics of North America, 5(1), 107-120.

Reilly, H.M., Martineau, J.K., Moran, A., \& Kennedy, H. (1995). Nutritional screening: Evaluation and implementation of a simple nutrition risk score. Clinical Nutrition 14, 269-273.

Rhodes, V., Johnson, M., \& McDaniel, R. (1995). Nausea, vomiting and retching: The management of the symptom experience. Seminars in Oncology Nursing, 11(4), 256-265.

Ross, B. (1990). Cancer's impact on the nutrition status of patients. In A. Bloch (Ed.), Nutrition Management of the Cancer Patient (pp. 11-13). Rockland, ML: Aspen.

Souba, W.W. (1997). Nutritional Support. The New England Journal of Medicine, 336(1), 41-48.

Sullivan, D.H. (1995). The role of nutrition in increased morbidity and mortality. Clinical Geriatric Medicine, 11, 661-674.

Szeluga, D.J., Stuart, R.K., Brookmeyer, R., Utermohlen, V., \& Santos, G.W. (1987). Nutritional support of bone marrow transplant recipients: A prospective randomized clinical trail comparing total parenteral nutrition to an enteral feeding program. Cancer Research, 47, 3309-3316.

Thomas, S. (1994). Gastrointestinal complications: Diarrhea and high gastric residuals. In B. Borlase, S. Bell, G. Blackburn, \& R. Forse (Eds.), Enteral Nutrition (pp. 188-192). New York: Chapman \& Hall.

Trujillo, E.B., \& Queen, P. (1994). Transition feeding. In B. Borlase, S. Bell, G. Blackburn, \& R. Forse (Eds.), Enteral Nutrition (pp. 107 - 114). New York: Chapman \& Hall.

Vaughn, T.L., \& McTierman, A. (1986). Diet in the etiology of cancer. Seminars in Oncology Nursing, 2(1), 3-13.

Weisdorf, S., Hofland, C., \& Sharp, H.L. (1984). Total parenteral nutrition in bone marrow transplantation: A clinical evaluation. Journal of Pediatric Gastroenterology and Nutrition, 3, 95100 .

Wickham, R.S., Rehwaldt, M., Kefer, C., Shott, S., Abbas, K., GlynnTucker, E., Potter, C., \& Blendowski, C. (1999). Taste changes experienced by patients receiving chemotherapy. Oncology Nursing Forum, 26(4), 697-710.

Wilmore, D.W., Smith R.J., \& Odwyer, S.T. (1988). The gut: A central organ after surgical stress. Surgery, 104, 917-928. 